\title{
Some remarks on new numerical estimations of the Rees-Sciama effect
}

\author{
Màrius Josep Fullana i Alfonso* \\ Insttitut de Matemàtica Multidisciplinària - Universitat Politècnica de València, València \\ E-mail: mfullana@mat.upv.es \\ Josep Vicent Arnau i Córdoba \\ Departament de Matemàtica Aplicada, Universitat de València. Burjassot, Spain \\ E-mail: arnauduv.es
}

\section{Robert J. Thacker}

Department of Astronomy and Physics, Saint Mary's University, Halifax, Nova Scotia, Canada

E-mail: thackereap.smu.ca

\section{Hugh M.P. Couchman}

Department of Physics and Astronomy, McMaster University, Hamilton, Ontario, Canada

E-mail: couchmanephysics.mcmaster.ca

\section{Diego P. Sáez Milán}

Departament d'Astronomia i Astrofísica, Universitat de València. Burjassot, Spain

E-mail: diego.saezeuv.es

In previous editions of Frontiers of Fundamental Physics Symposia, [1, 2, 3, 4, 5] we presented numerical computations of Cosmic Microwave Background (CMB) anisotropies at small angular scales (high $\ell$ 's). Our ray-tracing procedure was implemented in different N-body codes to calculate these anisotropies. A certain Particle-Mesh (PM) code and parallel Adaptative-ParticleParticle-Particle-Mesh (AP3M) Hydra codes have been used. This way we have been able to estimate weak lensing, Rees-Sciama (RS) and Sunyaev-Zel'dovich contributions to the CMB anisotropy. The use of a parallel AP3M code led to more accurate computations [6, 7], but further research is necessary. Almost a decade ago, we computed the RS effect by using a PM N-body code [8]. Here, some preliminary estimations of the RS effect, obtained with a parallel AP3M Hydra code, are presented. These results improve on those obtained with PM codes and, moreover, they are the starting point for a more detailed study of the RS anisotropy component.

Frontiers of Fundamental Physics 14

15-18 July 2014

Aix Marseille University (AMU) Saint-Charles Campus, Marseille, France

\footnotetext{
*Speaker.

$\dagger$ This work has been supported by the Spanish Ministry of Economía y Competitividad, MICINN-FEDER project FIS2012-33582. Calculations have been made using the Lluís Vives Computer of Universitat de València. We are greatful to the working team of the Servei d'Informàtica de la Universitat de València for useful help.
} 


\section{Introduction}

An appropriate ray-tracing procedure, through Particle-Mesh (PM) N-body simulations, was used in [8] to compute the Rees-Sciama (RS) contribution to the cosmic microwave background (CMB) anisotropy. Related talks - presented by the authors in previous FFP Symposium editionswere summarized in [1] and [2]. More recently, the same ray-tracing approach was combined with a parallel Adaptative Particle-Particle Particle-Mesh (AP3M) Hydra code, and we have used this methodology to estimate some nonlinear anisotropies such as the Sunyaev-Zel'dovich (SZ) and weak lensing (WL), see [6, 7]. Our ray-tracing method moves photons through a Universe that is generated by the simulation box allowing us to evaluate CMB foreground anisotropies (see below).

Here, the RS effect is studied again by using an AP3M N-body Hydra code, which may lead to resolutions better than those reachable with the simple PM code of reference [8]. The peculiar gravitational potential -necessary to estimate the RS anisotropy- is calculated and used at every time step of the N-body simulation, and CMB photons evolve under the action of this potential. Some advantages due to the use of an AP3M code -against the PM code of [8]- are pointed out. A more exhaustive study of the RS anisotropy -based on these advantages-is being performed and it will be presented elsewhere.

Although this paper is restricted to the RS effect, our main goal is the simultaneous study of RS, WL and SZ with the same simulations (AP3M Hydra code with baryons). Calculating all of the anisotropies self-consistently and simultaneously is clearly a challenging problem and we are working in it.

\section{Ray-tracing procedure}

Modern combined hydrodynamic and N-body codes can follow the evolution of dark matter and baryons inside cubic boxes at high efficiency. In the cosmological case, the box size must be large (at least a few hundreds of megaparsecs). Dark matter halos of different sizes are formed. There are galaxies and galaxy clusters with baryons inside these halos. Since the baryonic component is subdominant, only the dark matter distribution is actually important to estimate gravitational CMB anisotropies as RS and WL. The simulation box can be considered as a piece of the universe, which is uniformly covered by identical pieces of this type (at any time). Such a universe is periodic by construction. We consider CMB photons that move along appropriate null geodesics, whose directions are chosen in such a way that all the regions of the simulation box, crossed by the $\mathrm{CMB}$ photons, are statistically independent among them. This fact makes negligible any periodicity effect. Specific details about our method follow: For a box side $L=512 h^{-1} \mathrm{Mpc}$ and a direction defined by the spherical coordinates $\theta=73.34^{\circ}, \phi=16.96^{\circ}$, any photon, entering inside a certain box through the crossing point $P$, passes to the next box at point $Q$, and the distance, $\mathrm{D}$, between points $P$ and $Q$ is $\sim 104 h^{-1} \mathrm{Mpc}$; therefore, if the scales larger than $42 h^{-1} \mathrm{Mpc}$ are eliminated from the full potentials given by the AP3M code (only to perform RS calculations, but not to evolve dark matter), photons cross uncorrelated regions along successive boxes, which makes negligible periodicity effects in a periodic universe covered by boxes. Moreover, for the above direction, which is acceptable for our pourposes, $\mathrm{CMB}$ photons cross 16 boxes through independent regions from the initial redshift $z_{\text {in }} \sim 6$. $\left(\sim 8000 h^{-1} \mathrm{Mpc}\right)$ until present time. It is assumed that 
the RS signal is produced at $z<z_{i n}$, therefore the most extended lensed map we can build up is that corresponding to the image of a box face located -orthogonal to the line of sight- at $z=z_{\text {in }}$. The angular size of this image is close to $\sim 5^{\circ} \times 5^{\circ}$. Scales greater than $42 h^{-1} \mathrm{Mpc}$ are linear and the integrated Sachs-Wolfe effect they produce can be independently calculated (without N-body simulations), see [8].

Hereafter, $\vec{n}$ is the unit vector corresponding to the observation direction, $\Delta T / T$ is the relative temperature contrast, $\phi$ is the peculiar gravitational potential, $a$ is the scale factor, and whatever quantity $B$ may be, $B_{0}$ and $B_{e}$ stand for the $B$ values at present (observation) and emission (decoupling) times, respectively. In the flat universe under consideration we have chosen $a_{0}=1$.

Given a line of sight, an appropriate step must be selected to perform the integral involved in the following equation:

$$
\frac{\Delta T}{T}(\vec{n})=2 \int_{\lambda_{e}}^{\lambda_{0}} W(\lambda) \frac{\partial \phi}{\partial \lambda} d \lambda
$$

where $W(\lambda)=\left(\lambda_{e}-\lambda\right) / \lambda_{e}$. The variable $\lambda$ is

$$
\lambda(a)=H_{0}^{-1} \int_{a}^{1} \frac{d b}{\left(\Omega_{m 0} b+\Omega_{\Lambda} b^{4}\right)^{1 / 2}},
$$

$H_{0}$ and $\Omega_{\Lambda}$ being the Hubble constant and the vacuum density parameters, respectively. The matter density parameter is $\Omega_{m 0}=1-\Omega_{\Lambda}$. By using Eq. (2.1), the RS temperature contrast may be found for each direction involved in our ray tracing procedure, allowing maps of the RS effect being built up. The RS angular power spectrum ( $C_{\ell}$ coefficients) may be then calculated from these maps with appropriate methods (see [8] and references cited therein).

\section{Hydra Simulations}

All the simulations have been performed in a flat universe, whose Gaussian adiabatic scalar perturbations have a Zel'dovich post inflationary power spectrum. No vector and tensor modes are considered. We have chosen: $h=0.7, \Omega_{b}=0.046, \Omega_{d}=0.233, n_{s}=1, \tau=0.084$ and $\sigma_{8}=0.817$, where $h$ is the reduced Hubble constant, $\Omega_{b}\left(\Omega_{d}\right)$ is the baryon (dark matter) density parameter, $n_{s}$ is the spectral index of the scalar modes, $\tau$ is the reionization optical depth, and $\sigma_{8}$ is the fluctuation amplitude at $8 h^{-1} M p c$.

The initial conditions for Hydra simulations are calculated at redshift $z=50$. Initial displacements are calculated using the standard Zel'dovich approximation in the mildly nonlinear regime (up to $z=50$ ). A complete description of the parameters involved in our simulations may be found in [6]. In all the simulations considered in this paper, the box side length, the number of particles, and the initial redshift are $L_{b o x}=512 h^{-1} \mathrm{Mpc}, N_{p}=256^{3}$, and $z_{\text {in }}=6$, respectively. We allow parameters involved in the CMB anisotropy calculations to vary.

The simulations presented in this paper have been run by using the same Hydra code as in [6]. It is a parallel OpenMP-based implementation which uses the AP3M algorithm to compute gravitational forces within a box containing $N_{p}$ particles. In the AP3M algorithm a cubic "base" mesh with $N_{c}$ cells per side is supplemented by a series of refined-mesh P3M calculations to provide sub-mesh resolution. Gravitational softening is implemented using the S2 softening kernel which is remarkably similar in shape to the cubic spline softening kernel used in many treecodes (see 


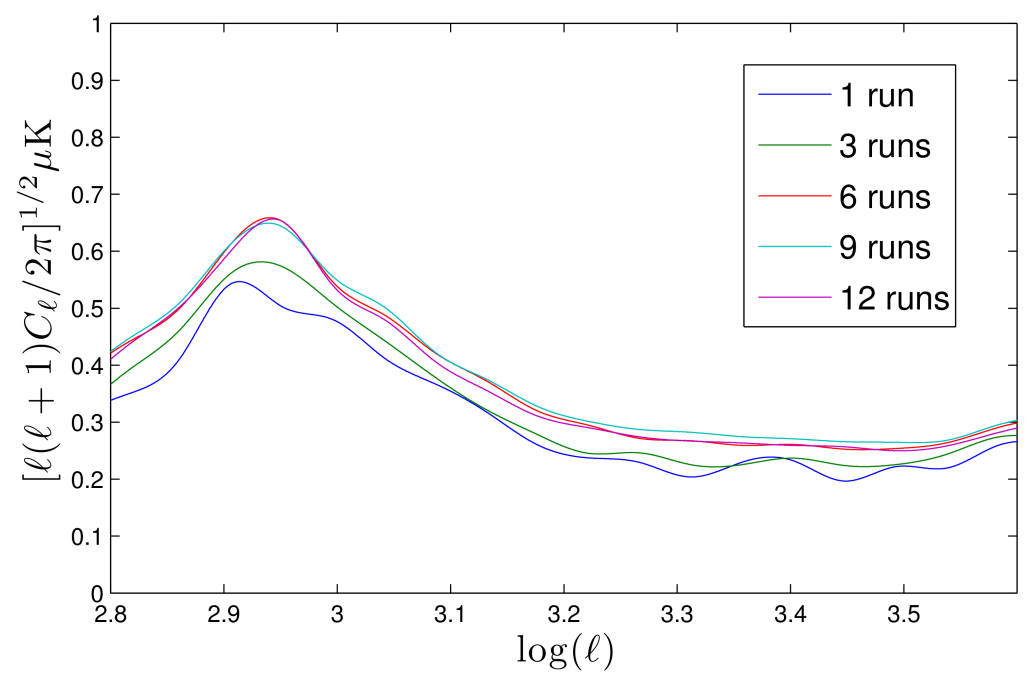

Figure 1: Averaged RS power spectra, in $\mu K$, as functions of $\log (\ell)$. Twelve simulations are considered. The 1-run spectrum (dark blue) is that having the smaller power. Averages corresponding to 3, 6, 9, and 12 runs are displayed

[6] for more details). The $\mathrm{S} 2$ softening used in the kernel is $2.34 \times S_{p}$ where $S_{p}$ is an equivalent Plummer softening length which we quote throughout the paper to enable a simple comparison to other work. The softening length is held constant in physical coordinates subject to the resolution not falling below 0.6 of the interparticle spacing at high redshift. This technique (hereafter called soft technique is widely applied and is a compromise between assuring that the potential energy of clusters does not evolve significantly at low redshift, while still ensuring structures and linear perturbations at high redshift are followed with reasonable accuracy. Hence, the soft technique must play an important role in this paper, where an integrated effect, due to the time derivative of the peculiar potential, $\phi$, is estimated.

\section{Results and Discussion}

Although the Integrated Sachs-Wolfe (ISW) and RS effects are both given by Eq. (2.1), the separation of these two anisotropy components is both possible and convenient. It is possible since it was proved in [2] that, at redshifts $z>3$, the integrated effect given by Eq. (2.1) correspond to a pure RS anisotropy (without ISW contribution) and, moreover, for $z<3$, almost all the ISW effect is produced by scales much greater than $60 \mathrm{Mpc}$, whose contributions to the integral in Eq. (2.1) have been suppressed (cutoff) to avoid periodicity effects. Hence, it may be stated that our maps of temperature contrasts -Hydra simulations plus ray tracing and cutoff- are an accurate representation of the RS effect, with negligible contamination from the ISW anisotropy. As it follows from Fig. 1, the RS signal is very small. It is dominated by other anisotropies for any angular scale ( $\ell$ value). A maximum power smaller than $1 \mu \mathrm{K}$ is reached for an $\ell$ value of the order of $10^{3}$. This effect is too small to be observable with current technologies. As in the case of the ISW anisotropy, future detection of the RS effect might be achieved by looking for correlations 


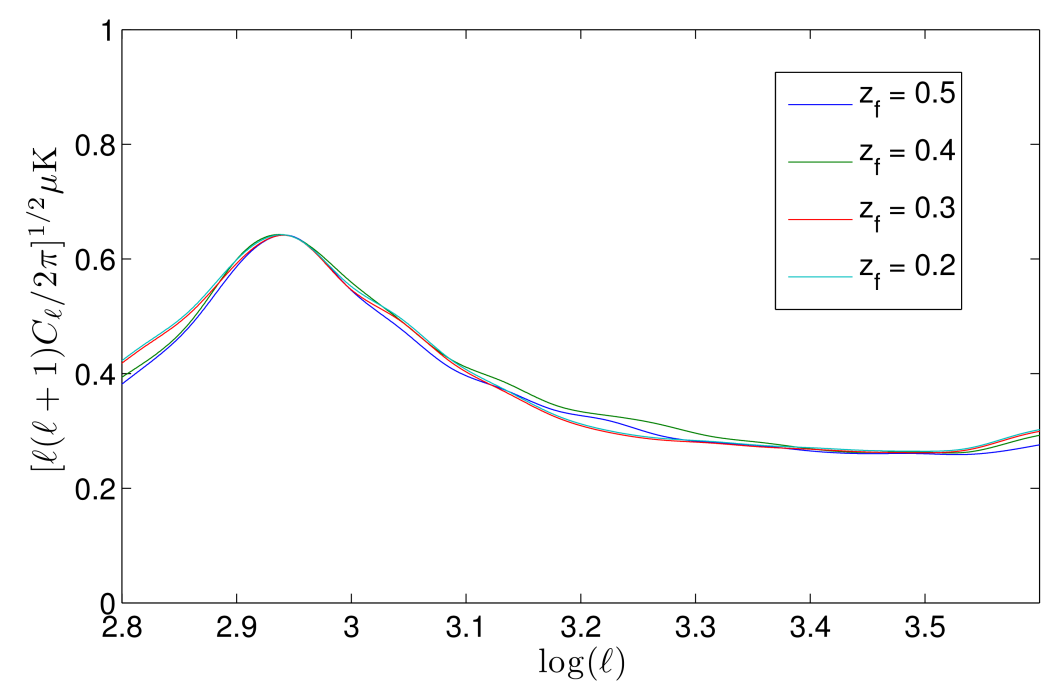

Figure 2: RS power spectra, in $\mu K$, as functions of $\log (\ell)$. Four values of the final redshift are considered.

between the observed distribution of the RS sources (clusters, superclusters an so on) and the CMB anisotropy maps. Any future method for RS detection would require both the knowledge of its isolated angular power spectrum and the right identification of its sources. This is being achieved with our simulations. A first step was given in [8].

Spectra similar to those of Figs. 1 and 2 were found in [8] by using a PM N-body code and the same ray tracing method as here. $512^{3}$ particles in boxes of $512 h^{-1} \mathrm{Mpc}$ were used to get a resolution of $\sim 350 \mathrm{kpc}$. Better numerical resolution and more accurate structure evolution requires a code with a more advanced solver, e.g., Hydra AP3M codes with a suitable soft technique and other appropriate elements. Thus the simulations presented here use the Hydra solver and dark matter particles, in which, particles have a mass of $8.8 \times 10^{11} M_{\odot}$, and a Plumber softening $S_{p}=6 h^{-1} k p c$ leads to a spatial resolution around $\sim 5 S_{p}=30 h^{-1} k p c$. This resolution is about ten times greater than the best resolution achieved with PM codes in [8].

The integration step -also called photon step $\Delta_{p s}$ - used to perform the integral in Eq. (2.1) is $\Delta_{p s}=15 h^{-1} k p c$. Finally, the number of null geodesics (lines of sight) is $256^{2}$ and the angular resolution of the RS maps is $1.17^{\prime}$; hence, the maximum $\ell$ value we may extract from our maps is $\ell_{\max } \simeq 9000$. One simulated map is not a large enough statistical sample to get the RS angular power spectrum. We therefore need to examine how many maps are necessary to produce a power spectrum that adequately samples over run to run variations. The answer to this question may be derived from Fig. 1, where one can see that the averaged spectra obtained with six, nine and twelve RS maps are very similar (convergence); hence, six runs are sufficient to get a good enough RS spectrum. In [8], where a PM code was used, around 30 time consuming runs were necessary to get comparable results. The new simulations presented lead to more accurate results using less particles, in the same size box, while also using less CPU time.

Any code simulating structure formation slows down as the redshift decreases and, moreover, nonlinear structures, as galaxy clusters, reach a rather stable phase at low redshifts. Hence, for 
these redshifts, the quantity $\partial \phi / \partial t$ and its contribution to the RS anisotropy [integral in Eq. (2.1)] are expected to be small. In order to verify this fact, six simulations have been run until four final redshifts, $z_{f}$, which are displayed in Fig. 2. The resulting angular power spectra are very similar among them. It is not possible to know if the slight spectra dispersion of Fig. 2 is due to the differences in $z_{f}$ or to the impact of using six simulations in the average (see Fig. 1). This means that -for RS estimations- the simulations may be stopped at a redshift of a few tenths, saving so a lot of CPU time being necessary to approach $z=0$ from $z_{f}$.

The impact of various parameters associated to the Hydra code and the ray tracing is being studied. We are paying particular attention on mass and space resolutions and the initial redshift. Results will be presented elsewhere.

\section{References}

[1] M.J. Fullana and D. Sáez, Making Maps of the Rees-Sciama Effect in Frontiers of Fundamental Physics: Proceedings of the Sixth International Symposium "Frontiers of Fundamental and Computational Physics". Sidharth, B.G., Honsell, F., de Angelis, A. (eds). Udine, Italy, September 26-29, 2004. Springer, The Netherlands, 115-122 (2006).

[2] M.J. Fullana and D. Sáez, Status of CMB Radiation in proceedings of Frontiers of Fundamental Physics: Eighth International Symposium FFP8. Sidharth, B.G., Alfonso-Faus, A., Fullana i Alfonso, M.J. (eds.). Madrid, Spain, 17-19 October, 2006. AIP Conference Proceedings 905, New York, USA, 13-22 (2007).

[3] M.J. Fullana, J.V. Arnau and D. Sáez, Weak Lensing on the CMB: Estimations Based on AP3M Simulations in proceedings of Frontiers of Fundamental and Computational Physics: 9th International Symposium. Sidharth, B.G., Honsell, F., Mansutti, O., Sreenivasan, K.R., Angelis, A. de (eds.). Udine and Trieste, Italy, January 7-9, 2008. AIP Conference Proceedings 1018, New York, USA, 80-85 (2008).

[4] M.J. Fullana, J.V. Arnau, R.J. Thacker, H.M.P. Couchman and D. Sáez, Observations and simulations of the CMB temperature anisotropy at very small angular scales in proceedings of Frontiers of Fundamental Physics: The Eleventh International Symposium. Kouneiher, J., Barbachoux, C., Masson, T., Vey, D. (eds.). Paris, France, July 6-9, 2010. AIP Conference Proceedings 1446, 252-260 (2012).

[5] M.J. Fullana, J.V. Arnau, R.J. Thacker, H.M.P. Couchman and D. Sáez, CMB anisotropy computations using Hydra gas code in proceedings of Frontiers of Fundamental Physics and Physics Education Research: 12th International Symposium. Sidharth, B.G., Michelini, Sanit, L. (eds.)Udine Italy, November 21-23, 2011. Spinger Proceedings in Physics 145, Switzerland, 189-196 (2014).

[6] M.J. Fullana, J.V. Arnau, R.J. Thacker, H.M.P. Couchman and D. Sáez, Estimating small angular scale Cosmic Microwave Background anisotropy with high-resolution N-body simulations: weak lensing. Astrophys. J. 712 (2010) 367.

[7] M.J. Fullana, J.V. Arnau, R.J. Thacker, H.M.P. Couchman and D. Sáez, A New Numerical Approach to Estimate the Sunyaev-Zel'dovich Effect in proceedings Progress in Mathematical Relativity, Gravitation and Cosmology. Garcia-Parrado, A. et al. (eds.). Guimarães, Portugal, September 3-7, 2012. Springer, The Netherlands, 277-282 (2014).

[8] N. Puchades, M.J. Fullana, J.V., Arnau and D. Sáez, On the Rees-Sciama effect: maps and statistics. Mon. Not. R. Astron. Soc. 370 (2006) 1849. 